\title{
Reconfinement, localization, and thermal monopoles in $S U(3)$ trace-deformed Yang-Mills theory
}

\author{
Claudio Bonati, ${ }^{1, *}$ Marco Cardinali $\odot,{ }^{1, \dagger}$ Massimo D’Elia, ${ }^{1, \star}$ Matteo Giordano ${ }^{2, \S}$ and Fabrizio Mazziotti $\odot^{3, \|}$ \\ ${ }^{1}$ Dipartimento di Fisica dell'Università di Pisa and INFN-Sezione di Pisa, \\ Largo Pontecorvo 3, I-56127 Pisa, Italy \\ ${ }^{2}$ ELTE Eötvös Loránd University, Institute for Theoretical Physics, \\ Pázmány Péter sétany 1/A, H-1117, Budapest, Hungary \\ ${ }^{3}$ Dipartimento di Ingegneria dell'Informazione, Università di Pisa, Via Caruso 16, I-56122 Pisa, Italy
}

(Received 20 January 2021; accepted 25 January 2021; published 15 February 2021)

\begin{abstract}
We study, by means of numerical lattice simulations, the properties of the reconfinement phase transition taking place in trace deformed $S U(3)$ Yang-Mills theory defined on $\mathbb{R}^{3} \times S^{1}$, in which center symmetry is recovered even for small compactification radii. We show, by means of a finite size scaling analysis, that the reconfinement phase transition is first order, like the usual $S U(3)$ thermal phase transition. We then investigate two different physical phenomena, which are known to characterize the standard confinement/ deconfinement phase transition, namely the condensation of thermal magnetic monopoles and the change in the localization properties of the eigenmodes of the Dirac operator. Regarding the latter, we show that the mobility edge signalling the Anderson-like transition in the Dirac spectrum vanishes as one enters the reconfined phase, as it happens in the standard confined phase. Thermal monopoles, instead, show a peculiar behavior: their density decreases going through reconfinement, at odds with the standard thermal theory; nonetheless, they condense at reconfinement, like at the usual confinement transition. The coincidence of monopole condensation and Dirac mode delocalization, even in a framework different from that of the standard confinement transition, suggests the existence of a strict link between them.
\end{abstract}

DOI: 10.1103/PhysRevD.103.034506

\section{INTRODUCTION}

Yang-Mills (YM) theories defined on the manifold $\mathbb{R}^{3} \times S^{1}$, where one of the directions is compactified, undergo a phase transition as soon as the length $L_{c}$ of the compactified direction becomes smaller than a critical length. If the compactified dimension is interpreted as the Euclidean time direction, then the length $L_{c}$ is just the inverse of the temperature $T$ of the system, and the phase transition is the well-known finite temperature deconfinement phase transition [1-4].

The deconfinement phase transition is associated with the spontaneous breaking of center symmetry [4-6], i.e., the invariance of the compactified theory under gauge transformations which are periodic up to an element of the

\footnotetext{
*claudio.bonati@unipi.it

†marco.cardinali@pi.infn.it

\#massimo.delia@unipi.it

\$giordano@bodri.elte.hu

"fabrizio.mazziotti@phd.unipi.it
}

Published by the American Physical Society under the terms of the Creative Commons Attribution 4.0 International license. Further distribution of this work must maintain attribution to the author(s) and the published article's title, journal citation, and DOI. Funded by SCOAP ${ }^{3}$. gauge group center along the compactified direction. The order parameter that signals the spontaneous breaking of center symmetry is the Polyakov loop, i.e., the holonomy of the gauge field along the compactified direction:

$$
P(\vec{x})=\mathcal{P} \exp \left(i g \int_{0}^{L_{c}} A_{4}(\vec{x}, \tau) \mathrm{d} \tau\right)
$$

where $\mathcal{P}$ denotes path ordering. Indeed, it is well known that $\operatorname{Tr} P$ transforms nontrivially under center transformations, and a nonzero value of $\langle\operatorname{Tr} P\rangle$ signals the finiteness of the free energy of an isolated static color charge, i.e., deconfinement.

The relation between center symmetry and other nonperturbative phenomena occurring in Yang-Mills theories, including the confining mechanism itself, is an open issue which still needs to be clarified. A useful theoretical tool, in this respect, is represented by trace deformation, which was introduced in Refs. [7,8]. For the case of the gauge group $S U(3)$ that will be studied in this paper, it consists in adding a term proportional to $|\operatorname{Tr} P(\vec{x})|^{2}$ to the $\mathrm{YM}$ action density. The rationale behind this choice is that such a term is invariant under center symmetry and, if its coefficient is chosen with the appropriate sign, it disfavors nonvanishing values of the trace of the Polyakov loop. Such a term 
compensates analogous ones that appear in the finite temperature effective potential [9], and its practical effect is that of increasing the deconfinement temperature or, equivalently, of reducing the critical length of the compactified direction. This opens the door to the possibility of studying confinement and other nonperturbative lowenergy properties in a finite temperature setup in which semiclassical methods can provide solid predictions [8].

The confinement mechanism at high temperature in the deformed theory, in what we will call the "reconfined phase," could clearly be different from the one of the original, undeformed YM theory: in the deformed case confinement is essentially enforced explicitly by a term in the Lagrangian, rather than emerging as a dynamical property of the theory. In spite of this, a number of lattice studies showed that the standard confined phase at $T=0$ in YM theory and the reconfined high-temperature phase of the deformed YM theory are remarkably similar, not only from a qualitative point of view but also quantitatively. Numerical simulations performed with gauge group $S U(N)$ both for $N=3[10]$ and $N=4$ [11] showed that the $\theta$ dependence in these two phases is the same, i.e., the values of the topological susceptibility and the coefficient of the $\theta^{4}$ term in the effective action are the same in standard $T=0 \mathrm{YM}$ theory and in the reconfined, finite- $T$ phase of the deformed YM theory. Very recently, it was shown that also the mass of the lowest glueball state is the same in these two phases [12].

In previous studies the main focus was on the investigation of the reconfined phase, far from the reconfining phase transition. In this paper we want to investigate, by lattice simulations, the properties of the deformed theory in the deconfined phase and around the transition, studying in particular the possible similarities or differences with respect to the standard deconfined/confined transition in YM theory. More precisely, after a finite size scaling analysis aimed at clarifying the location and the order of the phase transition, we will concentrate on two aspects that are tightly connected to the mechanisms of confinement and chiral symmetry breaking: monopole condensation and Dirac mode localization. As we will show, some aspects are similar to what happens around the usual confinement/ deconfinement transition, but not all of them; this could help to highlight some features of confinement and of the deformed YM theory.

The study of monopoles in lattice YM theories originates from the idea that color confinement can be a consequence of the condensation of magnetic degrees of freedom (dual superconductor scenario) [13,14]. Several strategies have been pursued in order to test this scenario, which go from the computation of the expectation value of a magnetically charged operator [15-23] to the extraction of the effective action of the monopoles [24,25]. Another possibility, which is the one we will follow in this work, is to study the behavior at the transition of thermal monopoles [26-37], i.e., the monopoles whose currents wrap around the compactified direction [26-28]. By studying the space-time configurations of these monopole currents it is possible to extract an effective chemical potential $\mu$ for the monopoles [32,37], whose condensation is signaled by the vanishing of $\mu$.

Systematic numerical investigations of the spectrum of the Dirac operator in lattice gauge theories appeared only recently, due to the high computational complexity of this task (see the reviews in Ref. [38] for results at zero temperature, and in Ref. [39] for the nonzero temperature case). The existence of a relation between the localization properties of the Dirac modes and the confinement properties of gauge theories is however by now a well established fact in a variety of models, including QCD with different fermionic discretizations [40-43], and other QCD-like theories [44-49]. In the deconfined phase in the trivial center sector of the Polyakov loop (i.e., the real sector), ${ }^{1}$ the lowest-lying Dirac modes are localized (i.e., their typical space-time size does not grow with the lattice volume), up to a temperature-dependent critical point in the spectrum, $\lambda_{c}$, known as "mobility edge." At the mobility edge, a continuous phase transition (Anderson transition) takes place in the spectrum [49-51], and Dirac modes become delocalized above $\lambda_{c}$. As the temperature is decreased, $\lambda_{c}$ approaches zero, and eventually vanishes in the confined phase, where all the low-lying Dirac modes are extended. In particular, this scenario has been tested numerically for $S U$ (3) YM using both the staggered Dirac operator [45] and the overlap Dirac operator [46]. It has been argued [52-54] that the presence or absence of localized modes is related to the different behavior of the Polyakov loop in the deconfined and confined phases of the theory. Since in the reconfined phase the Polyakov-loop expectation value vanishes, one would expect only delocalized Dirac modes. On the other hand, the reasons for its vanishing are different than in the usual confined phase, and it is worth checking whether this plays a role or not.

The paper is organized as follows. In Sec. II we describe the numerical setup and the lattice observables which are investigated in this study. Section III contains our numerical results: we first present a finite size scaling analysis of the transition between the deconfined and the reconfined phases of the deformed $S U(3)$ theory; then we study the properties of thermal monopoles and the localization properties of the low-lying Dirac modes around the transition. Finally in Sec. IV, we draw our conclusions and present some outlooks.

\section{NUMERICAL SETUP}

The action of trace-deformed $S U(3)$ YM reads

$$
S^{\text {def }}=S_{\mathrm{YM}}+h \int|\operatorname{Tr} P(\vec{x})|^{2} \mathrm{~d}^{3} x,
$$

\footnotetext{
${ }^{1}$ This is the center sector that would be selected by dynamical fermions. In the pure gauge case it is selected "by hand" when studying the localization properties of the Dirac modes.
} 
where $S_{\mathrm{YM}}$ is the standard YM action, $\vec{x}$ denotes a point on a hyperplane perpendicular to the compactified direction (i.e., the spatial coordinates of a point at a fixed time) and $h$ is the deformation coupling. Since each configuration is weighted in the Euclidean path integral by $e^{-S^{\text {def }}}$, positive values of the parameter $h$ disfavor nonvanishing values of $\operatorname{Tr} P(\vec{x})$.

A possible discretization of the action in Eq. (2) is the following:

$$
S^{\mathrm{def}}=S_{\mathrm{YM}}^{(\mathrm{W})}+h \sum_{\vec{n}}|\operatorname{Tr} P(\vec{n})|^{2},
$$

where $S_{\mathrm{YM}}^{(\mathrm{W})}$ is the usual Wilson action [55] with bare coupling $\beta$, and $\vec{n}$ is the lattice analogue of the variable $\vec{x}$ in Eq. (2). In our numerical simulations we used the lattice action Eq. (3), updating the spatial links with standard heatbath and overrelaxation algorithms [56-58], implemented for $S U(3)$ using the Cabibbo-Marinari procedure [59]; instead, temporal links were updated with a Metropolis algorithm [60], since they appear nonlinearly in the deformation term.

\section{A. Thermal monopoles}

The identification of Abelian magnetic monopoles in non-Abelian gauge theories goes through a procedure known as Abelian projection [61], which consists in a partial gauge fixing which breaks the gauge symmetry $S U(N)$ down to $U(1)^{N-1}$. In this work we used a variant of what is usually called maximal Abelian gauge (MAG). Standard MAG consists in the maximization of the sum of the squared moduli of the diagonal elements of all link matrices [62]: it is not affected by significant lattice artifacts (see, e.g., the discussion in Ref. [37]) and satisfies the Dirac quantization condition [63,64]. However, this procedure presents some drawbacks: for example, it leaves a residual permutation symmetry between the different $U(1)$ subgroups, thus preventing an unambiguous identification of the different monopole species when using more than two colors. For this reason a variation of the original MAG was put forward in Ref. [65], and we will follow the specific implementation proposed in Ref. [37], to which we refer for further details about the gauge fixing algorithm.

Once the gauge is fixed, it is possible to extract the Abelian components $a_{\mu}^{(i)}$ of each link (with $i=1, \ldots, N-1$ denoting the monopole species) and compute the associated DeGrand-Toussaint current $m_{\mu}^{(i)}$ [66], which is defined as

$$
m_{\mu}^{(i)}=\frac{1}{2 \pi} \epsilon_{\mu \nu \rho \sigma} \hat{\partial}_{\nu} \bar{\theta}_{\rho \sigma}^{(i)}
$$

where $\hat{\partial}_{\nu}$ is the forward lattice derivative, while $\bar{\theta}_{\rho \sigma}^{(i)}$ is derived from the plaquette $\theta_{\rho \sigma}^{(i)}$ computed using the Abelian fields $a_{\mu}^{(i)}$ as follows: $\theta_{\mu \nu}^{(i)}=\bar{\theta}_{\mu \nu}^{(i)}+2 \pi n_{\mu \nu}^{(i)}, \quad \bar{\theta}_{\mu \nu}^{(i)} \in[0,2 \pi), \quad n_{\mu \nu}^{(i)} \in \mathbb{Z}$.

In the lattice setup $n_{\mu \nu}^{(i)}$ is the analogue of the Dirac string piercing a plaquette, and this decomposition of $\theta_{\mu \nu}^{(i)}$ is needed to identify violations of the Abelian Bianchi identity (hence monopoles): the flux across any closed surface of the magnetic current constructed using just $\theta_{\mu \nu}^{(i)}$ would indeed identically vanish.

Due to the topological conservation law, $\partial_{\mu} m_{\mu}^{(i)}=0$, monopole currents form closed loops, and we identify thermal monopoles (antimonopoles) with the monopole currents which have a nontrivial positive (negative) winding number in the temporal direction. Such currents are interpreted as the paths of real (instead of virtual) magnetically charged quasiparticles populating the thermal medium [26-29]. Moreover, in analogy with the pathintegral formulation for a system of identical particles, currents which close after wrapping $k$ times around the thermal circle are interpreted as a system of $k$ thermal monopoles undergoing a cyclic permutation [32,37]: as for a system of identical bosons, the occurrence of such cycles is the relevant observable which can be used to investigate the possible condensation of the thermal particles [32].

In our study of thermal monopoles we measured the density of $k$-cycles $\rho_{k}$, defined as

$$
\rho_{k} \equiv \frac{N_{\text {wrap }, k}}{V_{s}}
$$

where $V_{s}=L^{3}$ is the spatial volume and $N_{\text {wrap }, k}$ is the number of monopole currents wrapping $k$ times around the thermal circle. From such quantities, the total density of thermal monopoles can be derived:

$$
\rho=\sum_{k} k \rho_{k} .
$$

\section{B. Dirac operator discretization and spectrum computation}

To investigate the localization properties of fermionic modes we employed the staggered discretization of the continuum Dirac operator, which is defined as follows:

$$
\begin{aligned}
& a D^{\mathrm{st}}(n, m) \\
& \quad=\frac{1}{2} \sum_{\mu=1}^{4} \eta_{\mu}(n)\left(U_{\mu}(n) \delta_{n+\hat{\mu}, m}-U_{\mu}(n-\hat{\mu})^{\dagger} \delta_{n-\hat{\mu}, m}\right),
\end{aligned}
$$

where $\eta_{\mu}(n)$ are the staggered phases, $U_{\mu}(n)$ is the link variable at site $n$ in direction $\mu$, and $a$ is the lattice spacing. Since $D^{\text {st }}$ is anti-Hermitian, its eigenvalues are purely imaginary. To fix the notation, we then write the eigenvalue equation as $a D^{\mathrm{st}} \psi_{n}=i a \lambda_{n} \psi_{n}, \lambda_{n} \in \mathbb{R}$, where $n$ labels the discrete modes of $D^{\text {st }}$ in a finite volume. Since $D^{\text {st }}$ 
anticommutes with $(-1)^{\sum_{\mu} n_{\mu}}$, its spectrum is symmetric with respect to $\lambda=0$, so that it suffices to consider $\lambda_{n} \geq 0$.

For each choice of the bare parameters we have computed the lowest part of the spectrum and the corresponding eigenvectors on a set of uncorrelated configurations, making use of the ARPACK routine [67]. Diagonalization was carried out after two steps of stout smearing on the gauge configuration, with stout parameter $\rho=0.15$ (see Ref. [68] for details).

\section{NUMERICAL RESULTS}

We have performed simulations for a fixed value of $N_{t}=6$ and $\beta=6.0$, changing only the deformation coupling $h$, or the spatial extent for finite size scaling purposes: this is sufficient for a first assessment of the properties of the reconfining transition, even if no continuum extrapolation is possible. Using the interpolation formula for the Sommer parameter $r_{0}$ of Ref. [69] and the phenomenological value $r_{0}=0.5 \mathrm{fm}$, we infer that our setup corresponds to a temperature $T=1 /\left(N_{t} a(\beta)\right) \simeq 360 \mathrm{MeV}$, meaning that at $h=0$ the system is in the deconfined phase: indeed, for $N_{t}=6$ the critical coupling for deconfinement is $\beta_{c}=5.8941(5)$ [70].

\section{A. The reconfinement transition}

In this section we study the reconfinement transition, by identifying the critical value $h_{c}$ such that for $h>h_{c}$ (at $\beta=6.0$ on $N_{t}=6$ lattices) the system is in the reconfined phase, and by investigating the order of the reconfinement transition.

To identify $h_{c}$ we monitor the behavior of the susceptibility of the modulus of the Polyakov loop,

$$
\chi_{P}=V_{s}\left(\left\langle|\operatorname{Tr} P|^{2}\right\rangle-\langle|\operatorname{Tr} P|\rangle^{2}\right),
$$

where $V_{s}=L^{3}$ is the spatial volume of the lattice and we used the shorthand

$$
\operatorname{Tr} P \equiv \frac{1}{V_{s}} \sum_{\vec{n}} \operatorname{Tr} P(\vec{n}) .
$$

For each spatial size $L$, data for $\chi_{P}$ have been produced using several values of $h$ and analyzed using the multiple histogram method [71]. The final results of this analysis are reported in Fig. 1: the susceptibility develops a peak that gets higher and narrower when increasing $L$, signaling the presence of a phase transition. Since the breaking pattern at reconfinement is the same $\mathbb{Z}_{3} \rightarrow$ Id of the standard deconfinement phase transition, the universality argument of Refs. [5,6] predicts a first order phase transition also in this case.

Finite size scaling (FSS) for first order phase transitions in a translation invariant setup predicts the following scaling behavior for the susceptibility of the order parameter:

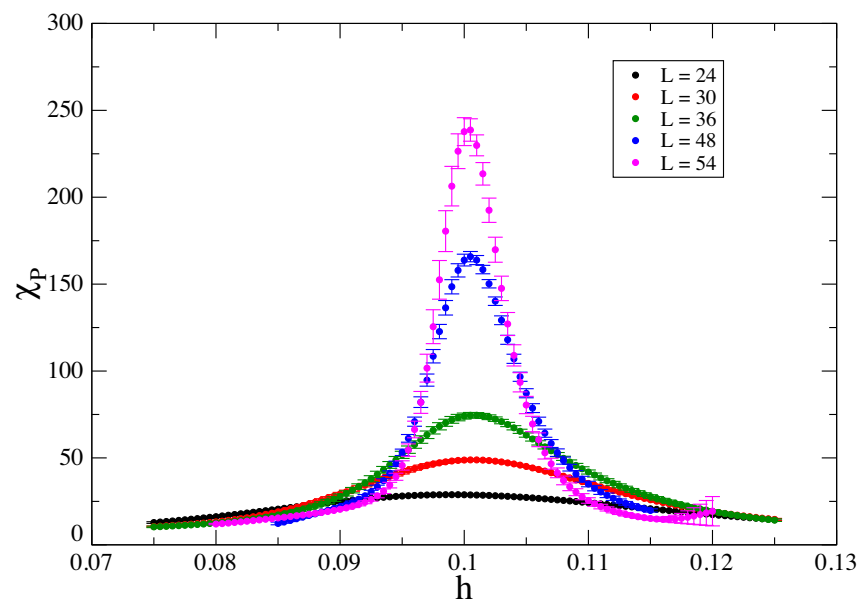

FIG. 1. Polyakov loop susceptibility $\chi_{P}$ as a function of $h$ for several spatial volumes, $\beta=6.0$ and $N_{t}=6$.

$$
\chi_{P}(h)=L^{\gamma / \nu} f\left(L^{1 / \nu}\left(h-h_{c}\right)\right),
$$

with effective critical exponents $\gamma=1$ and $\nu=1 / 3$ [72-75]. We thus first of all verified that the height of the peaks of the susceptibility scales as expected with the volume, fitting the maxima with the functional form

$$
\chi_{P}^{\max }=a L^{3 b},
$$

where $a$ and $b$ are fit parameters. The best fit value $b=0.98(3)$ is indeed consistent with the theoretical expectation $b=1$.

We then verified that the data are well described by the scaling ansatz in Eq. (11), using the critical exponents appropriate for a first order phase transition: the corresponding collapse plot is shown in Fig. 2 and fully supports the transition being first order. By varying $h_{c}$ in this scaling plot we estimated $h_{c}=0.100(2)$ : for $h_{c}$ outside this

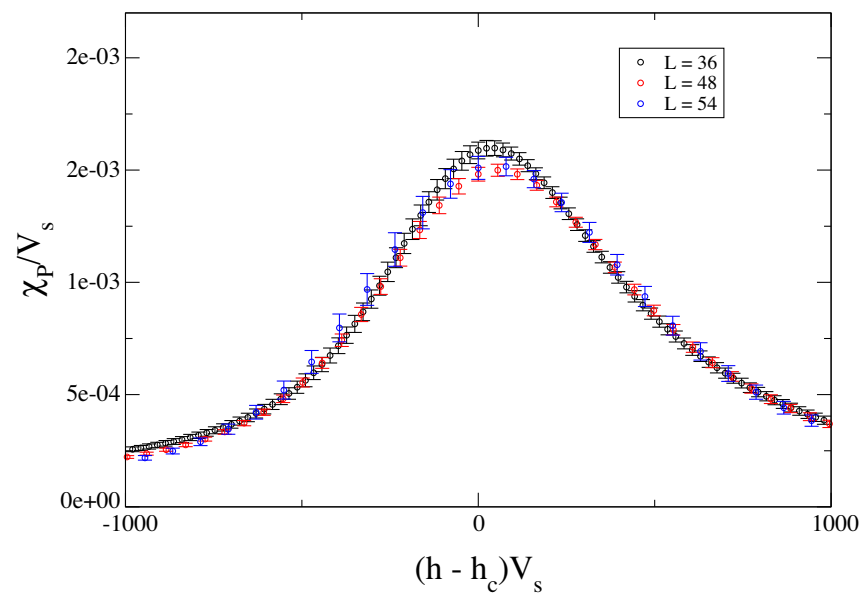

FIG. 2. Finite size scaling of the Polyakov loop susceptibility $\chi_{P}$ according to Eq. (11), after fixing $\nu=1 / 3, \gamma=1$ and $h_{c}=0.1$. 
interval the peaks of the data corresponding to $L=36$ and $L=54$ data sets become clearly separated from each other.

\section{B. Thermal monopole condensation}

Let us discuss first the results obtained for the total density of thermal monopoles, defined in Eq. (7). We consider, in particular, the dimensionless quantity $\rho / T^{3}$, which is reported as a function of $h$ in Fig. 3. The behavior of $\rho / T^{3}$ presents a significant difference with respect to what happens when approaching the usual confinement/ deconfinement transition [32,37]: in that case, $\rho / T^{3}$ grows as one moves from the deconfined towards the confined phase, and decreases at high $T$, where it approximately follows the behavior predicted by the perturbative analysis [76,77], $\rho / T^{3} \approx 1 /\left(\log \left(T / \Lambda_{\text {eff }}\right)\right)^{3}$, where $\Lambda_{\text {eff }}$ is some effective energy scale.

On the contrary, what we observe from Fig. 3 is that $\rho / T^{3}$ steeply decreases approaching the reconfined phase, has a big negative jump in correspondence of the first order phase transition, and then continues a slow decrease also in the reconfined phase. Results in the reconfined phase suggest the approach to a constant value in the large- $h$ limit; indeed, a best fit of results in the reconfined phase to a function

$$
\frac{\rho}{T^{3}}=\frac{\rho_{h=\infty}}{T^{3}}+A e^{-h / \bar{h}}
$$

returns $\rho_{h=\infty} / T^{3}=0.095(1)$ and $\bar{h}=0.20(5)$, with $\chi^{2} /$ d.o.f. $=2.8 / 2$.

The fact that $\rho / T^{3}$ decreases approaching reconfinement may seem at odds with the possibility that monopoles condense at the transition. However, this is emblematic of a

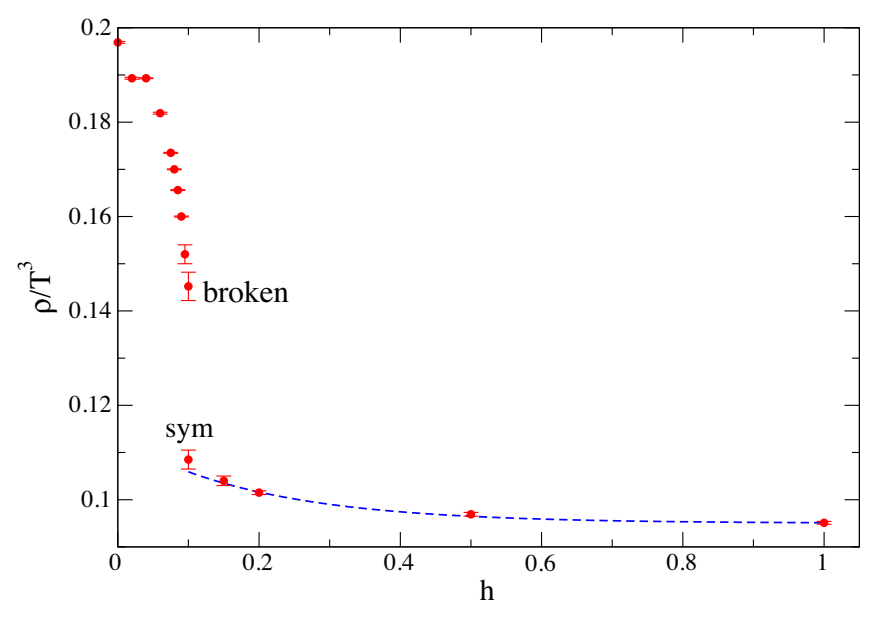

FIG. 3. $\rho / T^{3}$ computed using different values of the deformation parameter $h$ on a $54^{3} \times 6$ lattice at $\beta=6.0$. The two determinations reported for the transition point $h_{c}=0.1$ have been obtained by dividing the sample of configurations into two subsamples according to the realization of center symmetry (broken/unbroken). possible misinterpretation of the meaning of condensation, which is not necessarily related to an increase in the overall density, but rather to the appearance of a nonzero density of particles in the zero-momentum state. For a boson gas, such condensation is signalled in the path-integral formulation by a critical behavior of the density of $k$-cycles $\rho_{k}$ as a function of $k$, and this is indeed a possible criterion proposed to study thermal monopole condensation $[32,78]$. Therefore we now turn to this kind of analysis, which will finally show that, in fact, monopoles condense at $h_{c}$, in spite of the decrease of $\rho / T^{3}$.

In the path integral representation of the partition function of bosonic particles, quantum effects can be associated with particle paths undergoing a permutation around the thermal circle, hence to trajectories wrapping multiple times around the temporal direction, which represent the cycle decomposition of the corresponding permutation [79-82]. In particular, the densities $\rho_{k}$ are expected to behave as follows:

$$
\rho_{k} \propto \frac{e^{-\hat{\mu} k}}{k^{\alpha}}
$$

where $\hat{\mu} \equiv-\mu / T$, with $\mu$ the chemical potential, and $\alpha$ is a coefficient depending on the details of the system, for instance $\alpha=5 / 2$ for noninteracting nonrelativistic bosons. At high temperatures $\hat{\mu}$ is large and paths with multiple wrappings are rare, since one approaches Boltzmann statistics. On the contrary, $\hat{\mu}$ decreases at low temperatures and its vanishing signals the occurrence of a critical phenomenon like Bose-Einstein condensation (BEC). Once BEC is reached, $\hat{\mu}=0$ and $\rho_{k}$ follows a power-law behavior.

Results obtained for the ratio $\rho_{k} / \rho_{1}$ for different values of $h$ are reported in Fig. 4: it is already quite clear from this figure that the density of monopole trajectories having

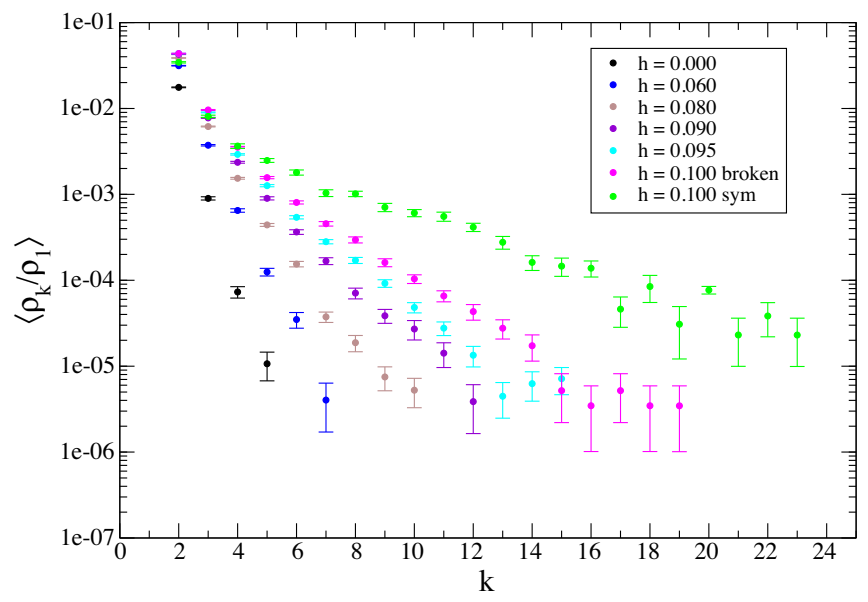

FIG. 4. $\left\langle\rho_{k} / \rho_{1}\right\rangle$ approaching the reconfined phase. The lattice used is a $54^{3} \times 6$ at $\beta=6.0$. 


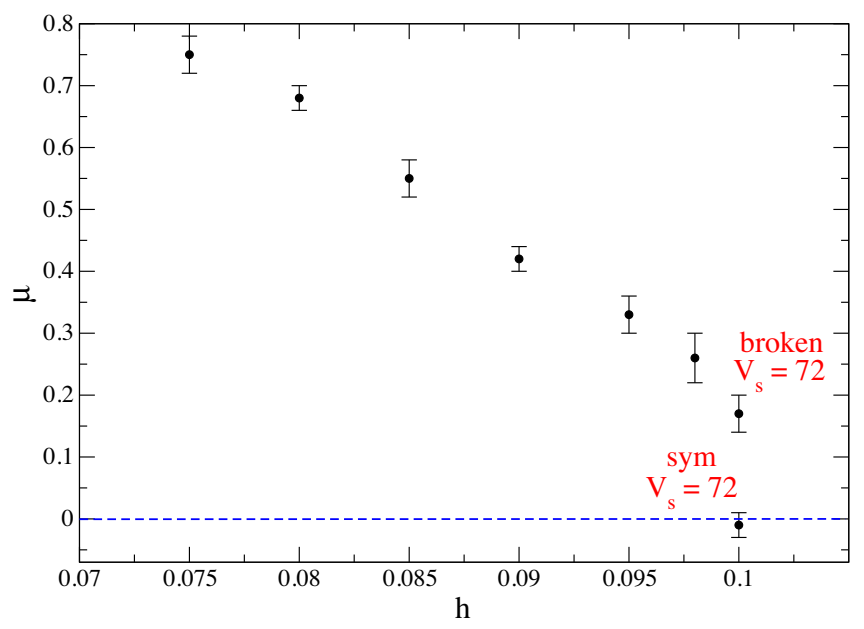

FIG. 5. The dimensionless chemical potential $\hat{\mu}$ extracted at different values of the deformation coupling $h$. The lattice used is a $54^{3} \times 6$ at $\beta=6.0$.

multiple wrappings increases as the coupling $h$ grows, i.e., approaching the reconfined phase.

Next we performed a best fit of $\rho_{k} / \rho_{1}$ to Eq. (14), in order to extract the chemical potential $\hat{\mu}$ as a function of $h$. As in Refs. [32,37], statistics are not precise enough to obtain an independent determination of $\alpha$, since different choices, including $\alpha=5 / 2$, lead to acceptable best fits; however, the analysis of Refs. [32,37] shows that this does not affect the determination of a possible point where $\hat{\mu}$ vanishes. Results obtained for $\hat{\mu}$ fixing $\alpha=5 / 2$ are shown in Fig. 5. The dimensionless chemical potential decreases as the system moves towards the reconfined phase, however it seems not to reach the value $\hat{\mu}=0$ at the critical $h_{c}$. This can still be compatible with a monopole condensation scenario if the chemical potential has a jump, rather than vanishing continuously, at $h_{c}$. This would be consistent with the presence of a first order transition, which would be stronger, at least for what concerns monopole condensation, than in the standard case of the thermal phase transition, where instead $\hat{\mu}$ does not show any appreciable jump at the transition [37]. In order to check this hypothesis, however, one should verify that the behavior of $\rho_{k}$ right in the reconfined phase is compatible with a powerlaw behavior, i.e., with a vanishing value of $\hat{\mu}$.

In order to clarify this point we performed simulations at $h=h_{c}$, dividing the configurations into two different subsamples: the "symmetric" configurations where center symmetry is unbroken (i.e., $\operatorname{Tr} P \simeq 0$ ), and the "broken" configurations where center symmetry is broken (i.e., $\operatorname{Tr} P \neq 0$ ); in order to make the division sharper and better defined, these additional simulations were performed adopting a larger spatial size, in particular a $72^{3} \times 6$ lattice. The increase in the relative occurrence of trajectories with a high number of wrappings in the symmetric configurations, clearly visible in Fig. 4, is suggestive of a sudden change in the behavior of $\rho_{k}$ as $h$ crosses the critical value. We have then fitted the two sets of configurations using Eq. (14) with $\alpha=5 / 2$. While a nonzero value of $\hat{\mu}$ is returned by the fit for the broken configurations, a value compatible with zero is obtained for the symmetric ones. This supports the monopole condensation scenario in the reconfined phase. It should however be noted that since the present quality of the data does not allow to determine the power-law exponent independently, other possibilities are not entirely excluded.

\section{Localization properties of Dirac modes}

In this section we study the localization properties of the eigenmodes of the Dirac operator on the lattice. As it is known from Refs. [45,46], in the deconfined phase of undeformed $S U(3)$ YM (i.e., at $h=0$ ) in the trivial center sector of the Polyakov loop, the lowest Dirac modes are localized in a finite spatial region of the lattice, for eigenvalues below a critical mobility edge, $\lambda_{c}$, in the spectrum. Higher modes, above the mobility edge, are instead delocalized on the whole space. Approaching the confined phase from higher temperatures, the spectral range where modes are localized shrinks, i.e., $\lambda_{c}$ decreases, eventually vanishing at a temperature compatible with the deconfinement temperature. Here we want instead to investigate what happens when the system approaches the reconfined phase in the trace-deformed theory, starting from the deconfined phase: using the same setup described above, we have studied the spectrum of the staggered Dirac operator in a range of deformation couplings $h$ starting from $h<h_{c}$ and reaching the transition region. Configurations were restricted to the trivial center sector, by multiplying the temporal links on the last time slice by the appropriate center element if necessary.

The simplest observable sensitive to the localization properties of the Dirac modes is the so-called participation ratio (PR), which essentially measures the fraction of lattice volume occupied by a mode. For our purposes it is convenient to use a gauge-invariant definition of the PR. We have then set

$$
\mathrm{PR}_{n}=\frac{1}{V_{s} N_{t}}\left[\sum_{\vec{n}, t}\left|\psi_{n}^{\dagger}(\vec{n}, t) \psi_{n}(\vec{n}, t)\right|^{2}\right]^{-1},
$$

where $\psi^{\dagger} \psi$ denotes the scalar product in color space. If modes in a given spectral range are localized, their PR (averaged over configurations) will tend to 0 as $1 / V_{s}$ as the volume is increased; if instead they are delocalized, their PR will tend to a constant. Equivalently, one can look at the average spatial "size" of the mode, $\mathrm{VPR} \equiv V_{s} \cdot \mathrm{PR}$, which will tend to a constant or diverge like $V_{s}$ for localized or delocalized modes, respectively.

In Fig. 6 we show the VPR of the low Dirac modes for different lattice setups. We have considered three values of the deformation coupling, namely $h=0.05,0.07,0.09$, which are less than the critical coupling $h_{c}$, and two spatial 

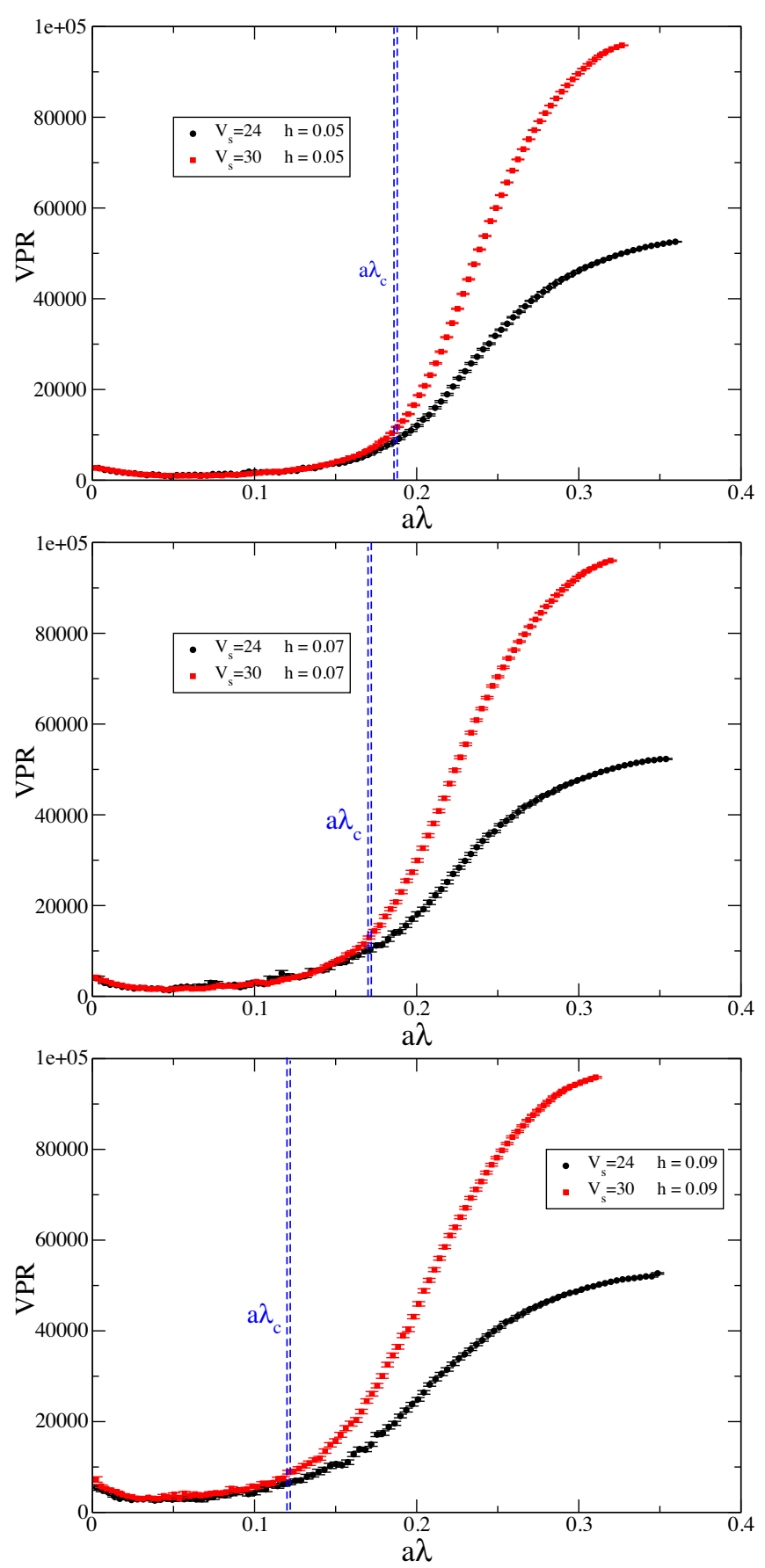

FIG. 6. Average VPR of the lowest staggered eigenmodes for different values of the deformation coupling $h$ below the critical one, and for two spatial volumes.

volumes $V_{s}=24^{3}, 30^{3}$. After dividing the spectrum in small bins, we averaged the VPR over configurations separately within each bin. In Fig. 6 one can clearly distinguish two regions. For the lowest modes the VPR does not change with $V_{s}$, indicating that they are localized. Higher up in the spectrum, instead, the VPR of the bulk modes grows with $V_{s}$. Assuming the scaling law $\langle\mathrm{VPR}\rangle_{\lambda} \sim C(\lambda) L^{\alpha(\lambda)}$, where $\langle\mathrm{VPR}\rangle_{\lambda}$ is the VPR averaged locally in the spectrum, and $\alpha$ is the fractal dimension of the corresponding modes, one can obtain $\alpha$ by comparing the two available volumes. For the bulk modes $\alpha \sim 3$, as expected for delocalized modes. This scenario is exactly the same found in nondeformed, deconfined YM [45].

It is clear from Fig. 6 that the region where modes are localized shrinks as $h$ approaches the critical value $h_{c}$. In order to make this statement more quantitative, we have identified the mobility edge $\lambda_{c}(h)$ by comparing the fractal dimension of the modes with the critical fractal dimension $\alpha_{c}=1.173_{-0.026}^{+0.032}$ found at the mobility edge in the unitary Anderson model [83]. The critical behavior at the mobility edge is in fact expected to be universal and determined only by the symmetry class of the system in the classification of random matrix theory, which for the staggered Dirac operator is the unitary one [84]. In Fig. 7 we show $\lambda_{c}(h)$ as obtained from $\lambda_{c}(h)=\alpha_{c}$ for the various choices of $h$. The tendency of $\lambda_{c}(h)$ to decrease as $h \rightarrow h_{c}$ is evident.

A look at the lowest modes of the staggered operator in the reconfined phase shows no evidence of localization. More precisely, we looked at the low modes on a few configurations at $h=0.11$ for $V_{s}=24^{3}, 30^{3}$, and found in the lowest bin $(a \lambda \leq 0.01) \mathrm{PR} \sim 0.24$ for $L=24$ and $\mathrm{PR} \sim 0.21$ for $L=30$. To investigate this issue further, we have generated configurations at the critical coupling $h_{c}=0.1$ and divided them in two subsamples, according to the fate of center symmetry, as we have done for the analysis of thermal monopoles. For both sets we have computed the PR on two different spatial volumes, namely $V_{s}=54^{3}$ and $V_{s}=72^{3}$. In Fig. 8 we show our results for the symmetric configurations, corresponding to the reconfined phase. The independence of the PR of the system size confirms the absence of localized modes in the reconfined phase, all the way down to $h_{c}$. Absence of localized modes

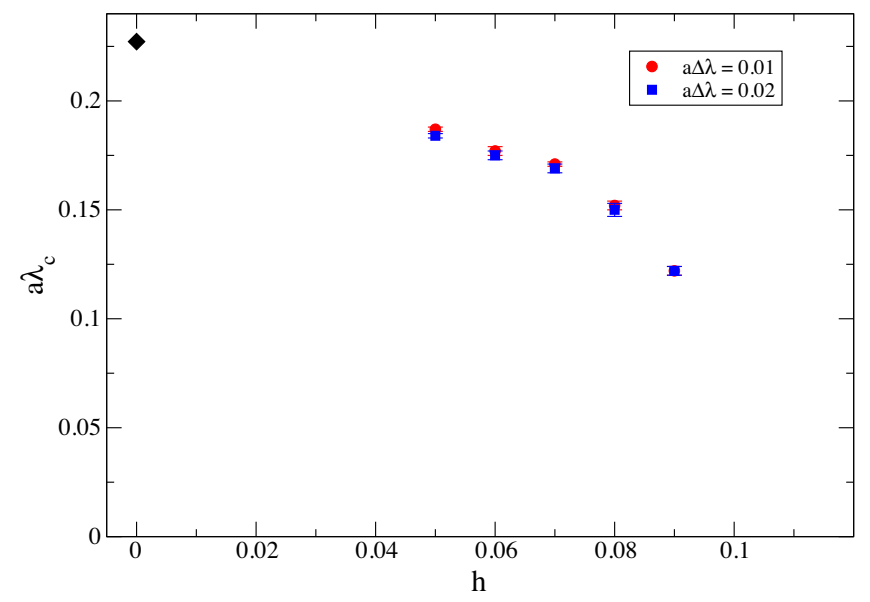

FIG. 7. Mobility edge, $\lambda_{c}$, as a function of the deformation parameter $h$. The two sets of points correspond to using a different bin size $\Delta \lambda$ in the extraction of $\alpha(\lambda)$ from the VPR. The black square is $\lambda_{c}(h=0)$ obtained by the authors of Ref. [45], and is shown for comparison. 


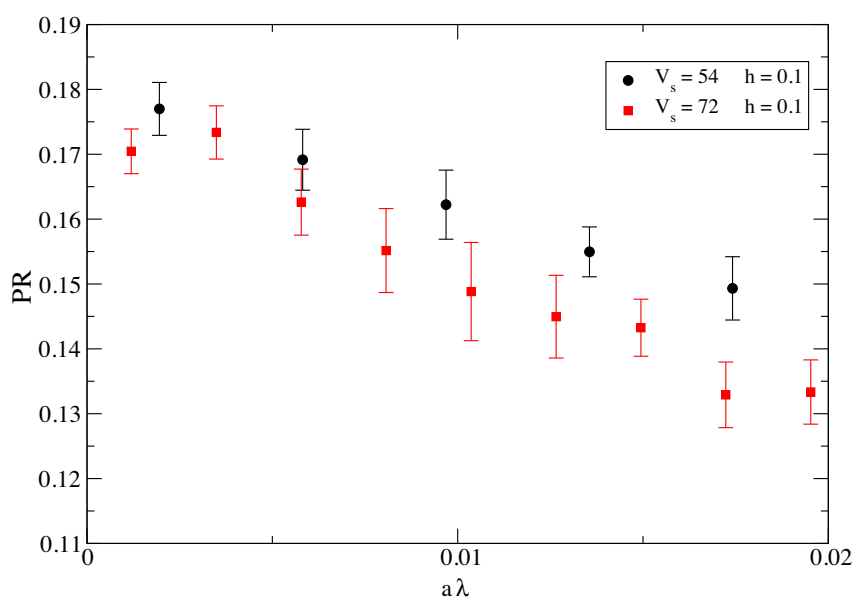

FIG. 8. Average PR of the lowest staggered eigenmodes on symmetric configurations at $h=h_{c}=0.1$.

in the reconfined phase can be expressed by setting $\lambda_{c}\left(h>h_{c}\right)=0$. It is not clear from Fig. 7 whether $\lambda_{c}$ approaches zero continuously or not as $h \rightarrow h_{c}$ : in analogy with what is observed for monopole condensation, it is reasonable to suppose that there is a jump also in this case. We would then expect to find localization, and so a nonzero mobility edge, in the broken configurations at $h=h_{c}$. Unfortunately, the analysis of these configurations still suffers from sizable finite-size effects for the available volumes, and we cannot make a conclusive statement.

\section{CONCLUSIONS}

In this paper we have studied the reconfinement phase transition of trace deformed $S U(3)$ YM theory by means of lattice simulations. In our analysis we have considered three aspects of the phase transition: (i) the order of the transition; (ii) the behavior of thermal monopoles; and (iii) the localization properties of Dirac eigenmodes. All results were obtained on a lattice with temporal size $N_{t}=6$ at $\beta=6.0$, corresponding to an extension in the compactified direction of approximately $(360 \mathrm{MeV})^{-1}(0.55 \mathrm{fm})$. The order of the transition was determined with a FSS study, which showed that the reconfinement phase transition is first order, exactly as the standard YM thermal deconfinement transition. This result is not surprising, because both in undeformed and deformed YM the symmetry breaking pattern is the same, i.e., $\mathbb{Z}_{3} \rightarrow$ Id. What the deformation does is just suppressing local fluctuations of the Polyakov loop (see Ref. [10]), forcing the order parameter $\langle\operatorname{Tr} P\rangle$ to be equal to zero. It would be interesting, in future studies, to extend this FSS analysis to the case of a smaller compactification length, in order to see how the critical value of the deformation coupling changes. Moreover, a detailed study of the order of the possible phase transition in trace deformed $S U(N)$ with $N>3$ would also be very interesting.
Some particular features emerged from the study of thermal monopoles. We have showed that their normalized density, $\rho / T^{3}$, decreases approaching the reconfinement transition and also beyond, reaching a plateau value in the large- $h$ limit. The fact that the trace deformation induces a decrease in the monopole density is not completely unexpected. Abelian magnetic monopoles are localized around points where two eigenvalues of the corresponding Higgs field vanish [61]: had we studied monopoles in the Polyakov gauge, a decrease in the monopole density would have been a consequence of the fact that the trace deformation induces a repulsion among the Polyakov loop eigenvalues. However, it is natural to expect that this might have an indirect effect also on monopoles defined in other Abelian projections, like MAG. In spite of the decreased density, thermal monopoles show a behavior compatible with a BEC-like condensation at reconfinement, as it happens at the standard confinement/deconfinement phase transition. It is interesting that there seems to be a significant jump in observables related to thermal monopoles at the reconfinement transition point: this is at odds with what is observed around the standard thermal transition, and might indicate that the transition is stronger in this case.

The localization properties of Dirac modes were studied using the staggered discretization of the Dirac operator. We found that the behavior of the low-lying modes at the reconfinement transition is similar to that observed at the usual confinement phase transition in the undeformed theory. While the system is in the deconfined phase in the trivial center sector, the lowest modes of the staggered Dirac operator are localized both at zero and nonzero deformation coupling, up to a critical point (mobility edge) in the spectrum. Bulk modes above the mobility edge are instead delocalized on the whole lattice. As the deformation coupling grows at fixed compactification length and the system moves towards the reconfined phase, the mobility edge decreases, and localized modes eventually disappear as the system crosses over into the reconfined phase. This is exactly what happens in standard (undeformed) YM, with the mobility edge decreasing when the temperature is decreased, and localized modes disappearing when crossing over to the usual confined phase [45]. It is not clear whether $\lambda_{c}$ vanishes continuously or discontinuously at the reconfinement transition: while a discontinuous behavior would be consistent with the first-order nature of the transition, and with what we observed for the thermal monopole observables, further studies are required to make a conclusive statement. In future studies one could also consider a different discretization of the Dirac operator, for example the overlap discretization, as it has been done in standard YM [46].

To summarize, we have demonstrated that two well established phenomena, which are known to characterize the confinement transition in $S U(3)$ gauge theory, characterize the reconfinement transition as well: condensation of 
thermal monopoles and delocalization of the lowest Dirac modes. This indicates that important physical features of the two transitions are similar, and at the same time establishes a stronger link between the two phenomena that we have analyzed. It is reasonable to hypothesize that the presence of thermal monopole trajectories in a gauge configuration has some reflection on the eigenmodes of the Dirac spectrum, and that the delocalization of thermal monopole trajectories, related to the appearance of larger and larger numbers of wrappings, could be related to the delocalization of the low-lying Dirac modes. On the other hand, the density of monopoles seems to have little bearing on it, as seen by contrasting the usual confinement transition and the reconfining one. This is surely something to be further investigated in future studies.

\section{ACKNOWLEDGMENTS}

We thank T. G. Kovács and R. Vig for useful discussions and for making their results available to us. Numerical simulations have been performed at the Scientific Computing Center at INFN-PISA and on the MARCONI machine at CINECA, based on the agreement between INFN and CINECA (under Projects No. INF19_npqcd, INF20_npqcd and IscraB Project No. IsB20_TDEDGE). M. G. was partially supported by the Nemzeti Kutatási, Fejlesztési és Innovációs Hivatal Grant No. KKP-126769.
[1] N. Cabibbo and G. Parisi, Phys. Lett. 59B, 67 (1975).

[2] A. M. Polyakov, Phys. Lett. 72B, 477 (1978).

[3] L. Susskind, Phys. Rev. D 20, 2610 (1979).

[4] L. D. McLerran and B. Svetitsky, Phys. Rev. D 24, 450 (1981).

[5] B. Svetitsky and L. G. Yaffe, Nucl. Phys. B210, 423 (1982).

[6] L. G. Yaffe and B. Svetitsky, Phys. Rev. D 26, 963 (1982).

[7] J. C. Myers and M. C. Ogilvie, Phys. Rev. D 77, 125030 (2008).

[8] M. Ünsal and L. G. Yaffe, Phys. Rev. D 78, 065035 (2008).

[9] D. J. Gross, R. D. Pisarski, and L. G. Yaffe, Rev. Mod. Phys. 53, 43 (1981).

[10] C. Bonati, M. Cardinali, and M. D’Elia, Phys. Rev. D 98, 054508 (2018).

[11] C. Bonati, M. Cardinali, M. D’Elia, and F. Mazziotti, Phys. Rev. D 101, 034508 (2020).

[12] A. Athenodorou, M. Cardinali, and M. D'Elia, arXiv: 2010.03618.

[13] G. 't Hooft, in High Energy Physics EPS International Conference, Palermo 1975, edited by A. Zichichi (Editrice Compositori, Bologna, 1976).

[14] S. Mandelstam, Phys. Rep. 23, 245 (1976).

[15] L. Del Debbio, A. Di Giacomo, G. Paffuti, and P. Pieri, Phys. Lett. B 355, 255 (1995).

[16] A. Di Giacomo and G. Paffuti, Phys. Rev. D 56, 6816 (1997).

[17] A. Di Giacomo, B. Lucini, L. Montesi, and G. Paffuti, Phys. Rev. D 61, 034503 (2000).

[18] A. Di Giacomo, B. Lucini, L. Montesi, and G. Paffuti, Phys. Rev. D 61, 034504 (2000).

[19] J. M. Carmona, M. D’Elia, A. Di Giacomo, B. Lucini, and G. Paffuti, Phys. Rev. D 64, 114507 (2001).

[20] J. M. Carmona, M. D’Elia, L. Del Debbio, A. Di Giacomo, B. Lucini, and G. Paffuti, Phys. Rev. D 66, 011503 (2002).

[21] M. D’Elia, A. Di Giacomo, B. Lucini, G. Paffuti, and C. Pica, Phys. Rev. D 71, 114502 (2005).

[22] C. Bonati, G. Cossu, M. D'Elia, and A. Di Giacomo, Phys. Rev. D 85, 065001 (2012).

[23] P. Cea and L. Cosmai, J. High Energy Phys. 11 (2001) 064.
[24] M. N. Chernodub, M. I. Polikarpov, and A. I. Veselov, Phys. Lett. B 399, 267 (1997).

[25] M. N. Chernodub, K. Ishiguro, and T. Suzuki, Phys. Rev. D 69, 094508 (2004).

[26] M. N. Chernodub and V. I. Zakharov, Phys. Rev. Lett. 98, 082002 (2007).

[27] M. N. Chernodub and V. I. Zakharov, arXiv:hep-ph/ 0702245.

[28] S. Ejiri, Phys. Lett. B 376, 163 (1996).

[29] A. D'Alessandro and M. D’Elia, Nucl. Phys. B799, 241 (2008).

[30] J. Liao and E. Shuryak, Phys. Rev. Lett. 101, 162302 (2008).

[31] C. Ratti and E. Shuryak, Phys. Rev. D 80, 034004 (2009).

[32] A. D'Alessandro, M. D'Elia, and E. V. Shuryak, Phys. Rev. D 81, 094501 (2010).

[33] M. N. Chernodub, A. D’Alessandro, M. D'Elia, and V. I. Zakharov, arXiv:0909.5441.

[34] V. G. Bornyakov and V. V. Braguta, Phys. Rev. D 84, 074502 (2011).

[35] V. G. Bornyakov and V. V. Braguta, Phys. Rev. D 85, 014502 (2012).

[36] V. V. Braguta and A. Y. Kotov, Phys. Rev. D 86, 014511 (2012).

[37] C. Bonati and M. D’Elia, Nucl. Phys. B877, 233 (2013).

[38] P. de Forcrand, AIP Conf. Proc. 892, 29 (2007).

[39] M. Giordano, T. G. Kovács, and F. Pittler, Int. J. Mod. Phys. A 29, 1445005 (2014).

[40] T. G. Kovács, Phys. Rev. Lett. 104, 031601 (2010).

[41] G. Cossu and S. Hashimoto, J. High Energy Phys. 06 (2016) 056.

[42] T. G. Kovács and F. Pittler, Phys. Rev. D 86, 114515 (2012).

[43] A. M. García-García and J. C. Osborn, Phys. Rev. D 75, 034503 (2007).

[44] A. M. García-García and J. C. Osborn, Nucl. Phys. A770, 141 (2006).

[45] T. G. Kovács and R. A. Vig, Phys. Rev. D 97, 014502 (2018). 
[46] R. A. Vig and T. G. Kovács, Phys. Rev. D 101, 094511 (2020).

[47] T. G. Kovács and F. Pittler, Phys. Rev. Lett. 105, 192001 (2010).

[48] M. Giordano, S. D. Katz, T. G. Kovács, and F. Pittler, J. High Energy Phys. 02 (2017) 055.

[49] M. Giordano, J. High Energy Phys. 05 (2019) 204.

[50] M. Giordano, T. G. Kovács, and F. Pittler, Phys. Rev. Lett. 112, 102002 (2014).

[51] L. Ujfalusi, M. Giordano, F. Pittler, T. G. Kovács, and I. Varga, Phys. Rev. D 92, 094513 (2015).

[52] F. Bruckmann, T. G. Kovács, and S. Schierenberg, Phys. Rev. D 84, 034505 (2011).

[53] M. Giordano, T. G. Kovács, and F. Pittler, J. High Energy Phys. 04 (2015) 112.

[54] M. Giordano, T. G. Kovács, and F. Pittler, J. High Energy Phys. 06 (2016) 007.

[55] K. G. Wilson, Phys. Rev. D 10, 2445 (1974).

[56] M. Creutz, Phys. Rev. D 21, 2308 (1980).

[57] A. D. Kennedy and B. J. Pendleton, Phys. Lett. 156B, 393 (1985).

[58] M. Creutz, Phys. Rev. D 36, 515 (1987).

[59] N. Cabibbo and E. Marinari, Phys. Lett. 119B, 387 (1982).

[60] N. Metropolis, A. W. Rosenbluth, M. N. Rosenbluth, A. H. Teller, and E. Teller, J. Chem. Phys. 21, 1087 (1953).

[61] G. 't Hooft, Nucl. Phys. B190, 455 (1981).

[62] F. Brandstater, U. J. Wiese, and G. Schierholz, Phys. Lett. B 272, 319 (1991).

[63] C. Bonati, A. Di Giacomo, L. Lepori, and F. Pucci, Phys. Rev. D 81, 085022 (2010).

[64] C. Bonati, A. Di Giacomo, and M. D'Elia, Phys. Rev. D 82, 094509 (2010).

[65] J. D. Stack, W. W. Tucker, and R. J. Wensley, Nucl. Phys. B639, 203 (2002).
[66] T. A. DeGrand and D. Toussaint, Phys. Rev. D 22, 2478 (1980).

[67] R. B. Lehoucq, D. C. Sorensen, and C. Yang, ARPACK Users' Guide: Solution of Large-Scale Eigenvalue Problems with Implicitly Restarted Arnoldi Methods (SIAM, Philadelphia, 1998).

[68] C. Morningstar and M. J. Peardon, Phys. Rev. D 69, 054501 (2004).

[69] S. Necco and R. Sommer, Nucl. Phys. B622, 328 (2002).

[70] J. Fingberg, U. M. Heller, and F. Karsch, Nucl. Phys. B392, 493 (1993).

[71] A. M. Ferrenberg and R. H. Swendsen, Phys. Rev. Lett. 63, 1195 (1989).

[72] M. E. Fisher and A. N. Berker, Phys. Rev. B 26, 2507 (1982).

[73] M. S. S. Challa, D. P. Landau, and K. Binder, Phys. Rev. B 34, 1841 (1986).

[74] K. Vollmayr, J. D. Reger, M. Scheucher, and K. Binder, Z. Phys. B 91, 113 (1993).

[75] J. Lee and J. M. Kosterlitz, Phys. Rev. B 43, 3265 (1991).

[76] J. Liao and E. Shuryak, Phys. Rev. C 75, 054907 (2007).

[77] P. Giovannangeli and C. P. Korthals Altes, Nucl. Phys. B608, 203 (2001).

[78] M. Cristoforetti and E. Shuryak, Phys. Rev. D 80, 054013 (2009).

[79] R. P. Feynman, Phys. Rev. 90, 1116 (1953).

[80] R. P. Feynman, Phys. Rev. 91, 1291 (1953).

[81] V. Elser, Ph.D. thesis, University of California, Berkeley, 1984.

[82] D. M. Ceperley, Rev. Mod. Phys. 67, 279 (1995).

[83] L. Ujfalusi and I. Varga, Phys. Rev. B 91, 184206 (2015).

[84] J. J. M. Verbaarschot and T. Wettig, Annu. Rev. Nucl. Part. Sci. 50, 343 (2000). 